\title{
Synthetic Guidelines for the Precision Engineering of Gold Nanoparticles
}

Jakub W. Trzciński, Luca Panariello, Maximilian O. Besenhard, Ye Yang, Asterios Gavriilidis, Stefan Guldin*

Department of Chemical Engineering, University College London, Torrington Place, London WC1E 7JE, U.K.; *s.guldin@ucl.ac.uk

\begin{abstract}
Gold nanoparticles (AuNPs) are one of the most studied nanomaterials with applications spanning from catalysis to biomedicine. While numerous chemical protocols exist that allow bespoke tailoring of chemical, physical and biological properties, their translation towards industrial-scale production remains a challenge. Batch synthesis often suffers from poor reproducibility and scalability, while emerging approaches, such as continuous flow synthesis, are not widely implemented in research labs. In this review, we provide a critical overview over recent developments in the field of AuNP synthesis and provide synthetic guidelines for precision engineering of nanoparticle properties.
\end{abstract}

\section{Introduction}

After decades of intensive research, gold nanoparticles (AuNPs) now offer opportunities in a plethora of applications, ranging from the ultrasensitive detection of biomarkers in point-of-care systems [1], e.g. for naked eye ELISA [2] and lateral flow immunoassays [3], to environmental test kits [4], antimicrobial coatings [5], photovoltaics and other energy transfer systems [6]. AuNPs have been widely studied for specific interaction with biomolecules [7], as drug sensors [8] and antimicrobial agents against bacteria [9] and viruses [10]. The particle applicability typically relies on the tailoring of distinct properties, such as the surface plasmon resonance, assembly into more complex lattices [11], catalytic features [12] or biological activity [13], [14]. AuNP-based products are meanwhile entering the commercial market, with a number of startup companies and small/medium-sized enterprises offering unique solutions based on gold nanotechnology platforms [15]. We note that while out of scope herein, further implications on the widespread use of gold nanoparticles have to be considered, including the availability of resources and the environmental risk post-use [16].

Physical and chemical properties of AuNPs are strongly related to the size and shape of the metallic core as well as the displayed surface chemistry [17]. For example, in a range from sub to hundreds of $\mathrm{nm}$, smaller nanoparticles exhibit a higher cell uptake than larger ones [18]. The interaction of AuNPs with their environment is often dominated by the chemical composition and charge of the NP surface, often defined by an organic ligand shell [19]. Furthermore, anisotropy in shape and surface functionalisation plays an important role in many biomedical applications [20].

The achievable precision towards control over size, shape and surface chemistry is strongly dependent on the synthetic protocol [21], [22]. Most common manufacturing routes rely on solution-based bottom-up syntheses from chemical precursors but alternative routes exist via photochemical, electrochemical, or templating principles. Despite the extensive body of research, challenges remain on the synthetic engineering of suitable protocols that allow AuNP production with precise control over the structural properties, low dispersity and high yield as well as short synthesis time and low synthesis costs, all of which at high reproducibility across laboratories [23]. Herein, we review the available synthetic toolbox and critically compare suitable batch and continuous flow protocols towards implementation in scale-up nanomaterial engineering. 


\section{Synthesis in batch}

The first synthesis of colloidal gold particles dates back to 1857 when Sir Michael Faraday reduced gold chloride by phosphorus in an aqueous solution. As a milestone, this seminal experiment guided most of the colloidal synthetic methods that followed: reducing solvated gold salt in the presence of a surface passivating agent to prevent aggregation. A brief overview of some of the most common synthetic concepts for AuNP synthesis is shown in Figure 1 alongside a comparison of precursors, reducing agent, passivate ligands and resulting size range displayed in Table 1.

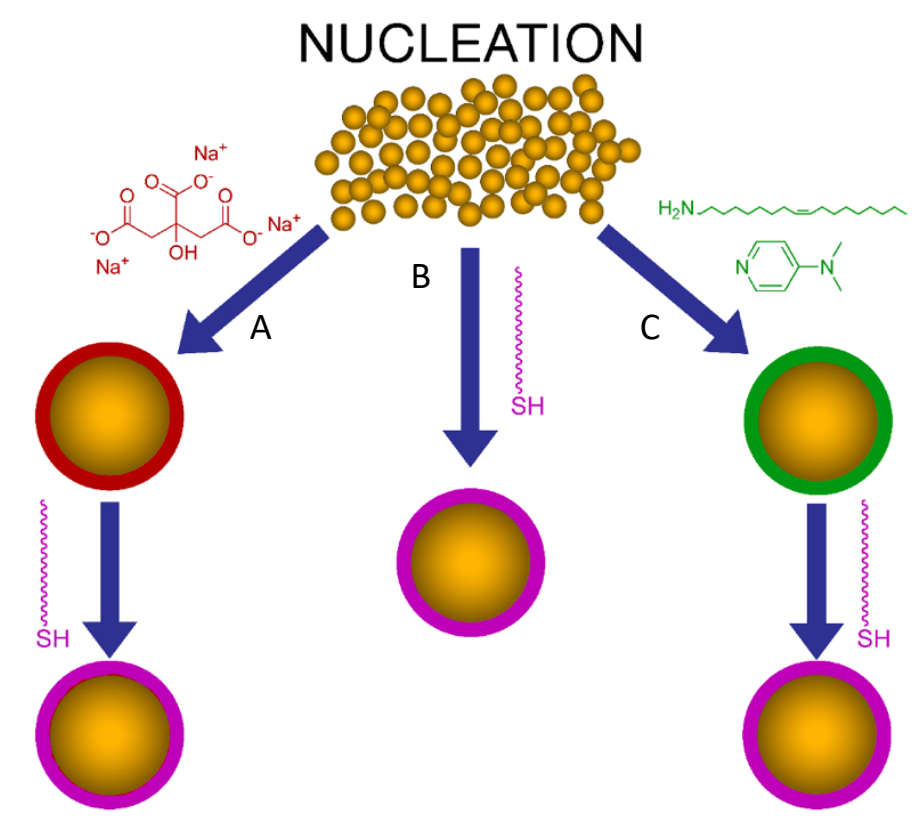

Figure 1. Schematic of the most common synthetic routes for: A) Charge-stabilized particles prepared by the Turkevich method using trisodium citrate and further exchange with a thiol, B) One-step synthesis with thiol capping directly after gold reduction, C) Two-step synthesis using labile ligand and subsequent ligand exchange with a thiol.

\begin{tabular}{|c|c|c|c|c|}
\hline $\begin{array}{c}\text { Gold } \\
\text { Precursor }\end{array}$ & Reducing agent & Passivating agent & Size [nm] & References \\
\hline $\mathrm{HAuCl}_{4}$ & Trisodium citrate & Trisodium citrate & $5-150$ & [24], [25] \\
\hline $\mathrm{HAuCl}_{4}$ & $\mathrm{NaBH}_{4}$ & TOABr, thiols & $1,5-5$ & [26], [27] \\
\hline$\left(\mathrm{Ph}_{3} \mathrm{P}\right) \mathrm{AuCl}$ & $\mathrm{NaBH}_{4}$ & Thiols & $1-8$ & [28] \\
\hline $\mathrm{HAuCl}_{4}$ & TBAB & Oleylamine & $2-6$ & [29] \\
\hline $\mathrm{HAuCl}_{4}$ & $\mathrm{NaBH}_{4}, \mathrm{CO}, \mathrm{GSH}$ & Thiols & $0,6-1,2$ & {$[30],[31],[32]$} \\
\hline
\end{tabular}

Table 1. Comparison of precursors, reducing agent and passivate ligands and resulting size range in some of the most common synthetic protocols for gold nanoparticle preparation.

Some of the most common AuNP synthesis routes are based on the reduction of $\mathrm{HAuCl}_{4}$ as gold precursor by sodium citrate, with seminal work by Turkevich and Frens. Notably, sodium citrate may act as reducing agent, stabilising agent and $\mathrm{pH}$ mediator, offering a versatile platform for optimisation [24]. Nevertheless, several inherent drawbacks remain, including the low yield of batch reactions as well as difficult access to monodisperse AuNPs below $10 \mathrm{~nm}$ in diameter. The latter challenge may be overcome by a synergistic interplay of sodium citrate and tannic acid, which offers a route to 3.5 to 10 $\mathrm{nm}$-sized citrate-stabilised particles with narrow size distribution [33]. Another area of research is the so-called seeds-mediated growth technique, where sub $10 \mathrm{~nm}$ gold seeds serve for subsequent particle growth [25]. This process, enabling gold reduction and inhibition of secondary nucleation, may be kinetically controlled by adjusting $\mathrm{pH}$, temperature and gold precursor to seeds concentration ratio [34]. The seed growth provides a versatile and reliable chemical route to the synthesis of uniform 
particles up to $200 \mathrm{~nm}$ as well as anisotropic shapes. In this context, Mirkin and co-worker established that the formation of anisotropic nanoparticles is strictly dictated by the shape, size and the crystalline structure of the seeds [35]. Iterative seed refinement by oxidative dissolution and reductive growth enabled them to create different shapes from an identical seed source (Figure 2).

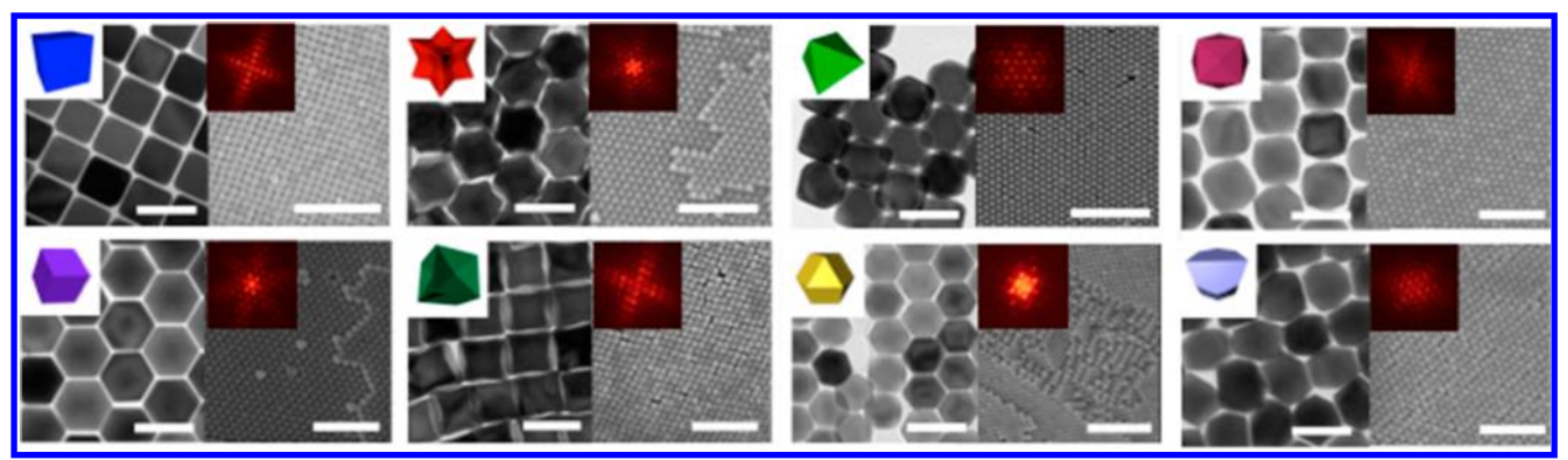

Figure 2. Relationship of AuNP seed and final shape, as manipulated by iterative oxidative dissolution and reductive growth from a single seed source. Reprinted with permission from reference [35]. Copyright 2014 American Chemical Society.

Beyond citrate-mediated electrostatic stabilisation of the AuNP surface, thiol-based ligands reliably passivate the gold core and constitute an extremely broad molecular platform for surface functionalisation, enabling the attachment of nucleic acids, proteins, peptides, lipids and a multitude of other molecules via covalent or non-covalent conjugation [36]. Several protocols offer direct access to thiol-capped AuNPs, most notably the two-phase [26] and single-phase synthesis by Brust et al [27]. and a single-phase synthesis introduced by Stucky and co-workers [28]. The former are most effective at synthesising 1-4 nm sized AuNP, the latter extends the achievable size range to $8 \mathrm{~nm}$ with improved size uniformity by the use of amine-borane complexes (TBAB) as reducing agent [29]. While the direct synthesis of thiol-protected AuNPs offers larger yield, shorter preparation times and smaller core diameters, the protocols are generally limited to organic solvents and non-polar thiol ligands. The replacement of the thiol capping layer via thiol-for-thiol ligand exchange is generally challenging due to high affinity of the gold-thiol bond [37].

To this end, the synthesis of AuNPs with a labile ligand as intermediate capping agent, followed by a ligand-for-thiol exchange provides a broad platform for AuNP synthesis with target functionality. Promising routes include the use of phosphines [38], 4-(N,N-dimethylamino)pyridine (DMAP) [39] and tetraoctylammonium bromide ( $\mathrm{TOABr}$ ) [27],[40]. In recent years, aliphatic amines including oleylamine have attracted particular interest [41]. In fact, we have recently shown that the use of oleylamine as stabilising agent enables the decoupled control of AuNP size and surface composition, with the former being controlled by the reaction temperature in the initial synthesis and the latter by the subsequent exchange with prescribed thiol ligand mixtures (Figure 3) [42]. 
(a)

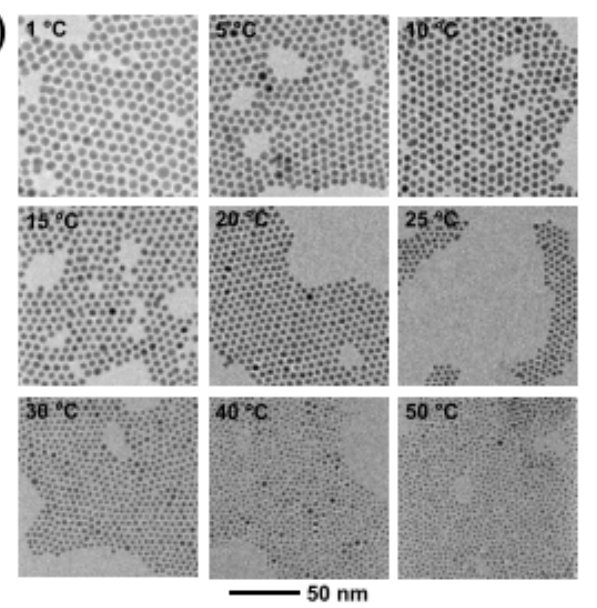

(b)

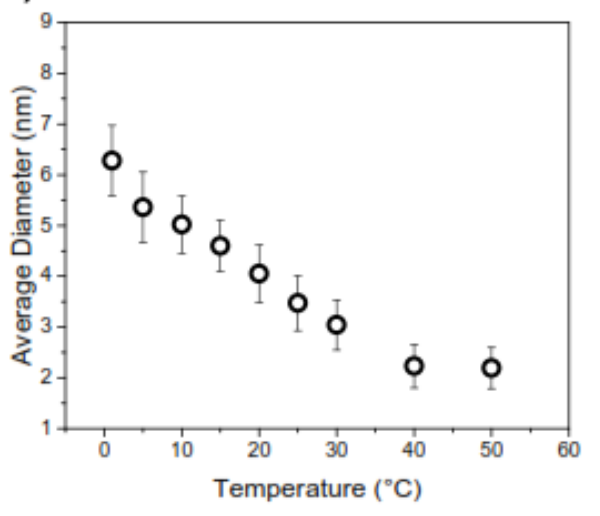

Figure 3. Size control offered by temperature manipulation in oleylamine-based AuNP synthesis. a) Representative TEM images for different reaction temperatures. b) Average size and standard deviation. Note that this approach offers a decoupled control of size and surface composition by a subsequent thiol-for-oleylamine ligand exchange. Reprinted with permission from reference [42] under Creative Commons CC-BY license. Copyright 2018 American Chemical Society.

In recent years, atomically precise gold clusters $\left(\mathrm{Au}_{10}, \mathrm{Au}_{15}, \mathrm{Au}_{18}, \mathrm{Au}_{25}\right)$ have emerged as a particular promising nanoparticle platform [32], with distinct characteristics related to their luminescence [30] and renal clearance [43]. The formation of stable, atomically sub-1nm clusters can be effectively realised by $\mathrm{Au}(\mathrm{III})$ reduction using either $\mathrm{NaBH}_{4}, \mathrm{CO}$, glutathione (GSH) or proteins in the presence of thiol molecules [32].

While traditional bottom-up synthesis of gold nanoparticles in batch provide fast and scalable pathways for AuNP production (up to gram scale), protocols are suffering from low batch-to-batch reproducibility and limited synthetic yield [23]. The intrinsic kinetics on the mixing of gold ions with the reducing agent defines the final product, rather than the thermodynamics [44]. Thus, concentration gradients generated by ineffective mixing can lead to the formation of nuclei with variable size, which afterwards causes polydisperse particle size distribution [45]. In addition, ineffective decoupling of nucleation and growth, i.e. the continued nucleation during particle growth, promotes dispersity in size and shape [46]. To this end, Besenhard et al. demonstrated the influence of batch mixing of gold precursor $\left(\mathrm{HAuCl}_{4}\right)$ and reducing reagent (trisodium citrate) on particle size and dispersity using a fix feed 5:1 ratio (citrate/tetrachloroauric acid) [47]. In contrast to previous reports, smaller nanoparticles with reduced polydispersity were obtained by at-once addition rather than dropwise injection. This work highlights the importance not only of the molar ratio between reagents but mixing conditions and the order of reagent addition into the reaction.

\section{Synthesis in flow reactors}

Flow micro- and millireactors offer advantages over traditional batch reactors in the synthesis of nanomaterials. Their small characteristic dimensions decrease the diffusion paths, allowing faster mixing, while their high surface-to-volume ratio enhances heat transfer rates. These features facilitate tight control of critical process parameters. Furthermore, continuous operation yields product volumes significantly exceeding the reactor volume. Over the last decade, there has been an increasing number of reports utilizing flow reactors for the synthesis of gold nanoparticles of varying shape, size and surface functionality. A plethora of reactor designs was employed to-date, including radial interdigitated mixers, split-and-recombine mixers, channels with butterfly structures, Y-shape pulsating mixers, coaxial flow mixers and coiled flow inverters [48]. 
Many flow synthesis studies claim that, due to fast mixing and enhanced heat and mass transfer, one can synthesize smaller particles in flow than in batch, and/or flow reactors lead to faster reaction kinetics. We note that this applies only to a limited number of cases, e.g. for reactions where the mixing time is comparable to the reaction time, or when the synthesis is mass-transfer-limited. Hence, the shorter reaction times associated with flow syntheses is often related to unoptimized batch protocols [49]. In this regard, flow reactors may inform and benefit the study of nanomaterials synthesis kinetics. At the same time, the study of the synthesis kinetics could render the translation of batch protocols to flow an easier task [39]. This was recently demonstrated by Panariello et al. who translated an Au nanoparticle synthesis to flow with minimal effort [50]. Batch studies provided the relevant kinetic information on precursor conversion, which enabled the design of a flow reactor with throughput 20 $\mathrm{mL} / \mathrm{h}(\sim 1 \mathrm{mgAu} / \mathrm{h})$ and a production volume limited only by the syringes used. Full exploitation of kinetic data can though only be achieved through the development of theoretical approaches that are able to utilise such information. Population balance modelling offer valuable information at limited computational cost, which can be combined with classical reactor design methodologies in order to design flow reactors synthesizing nanomaterials [51]. For gold nanoparticles, only few works on population balance modelling are available in the literature [52], [53].

Reproducibility is one of the main incentives of flow reactors, derived from increased control over experimental conditions and reduction of human intervention during the synthesis [50], [54], [55],. This can be further enhanced by improved process understanding and quality control achieved by the integration of online analysis, e.g. by the online use of SAXS/WAXS and UV-Vis [56], [57], [58]. Several techniques have been integrated in line, such as small angle X-ray scattering/wide angle X-ray scattering (SAXS/WAXS), extended X-ray absorption fine structure (EXAFS), and UV-Vis spectroscopy. The implementation in flow of new techniques, such as liquid-phase transmission electron microscopy (TEM) can bring significant advancement to the understanding of particle synthesis mechanism. Further progress in the field of process and quality control requires the integration of techniques, which allow monitoring of both size distribution and particle concentration. While this may currently be achieved by SAXS, challenges remain for more widely accessible techniques, including a relatively slow acquisition time for differential centrifugal sedimentation (DCS), unreliability for multimodal or wide size distributions for dynamic light scattering (DLS) and the relatively large limit of detection ( 10 $\mathrm{nm}$ ) for nanoparticle tracking analysis (NTA). UV-Vis can help in this direction, though the interpretation of UV-Vis spectra is not straightforward and requires a priori knowledge to obtain reliable results (e.g. particle size and surface coating) to determine concentration of spherical gold nanoparticles [59]. Atomic emission spectroscopy (AES) may provide yield values with no a priori knowledge of the analysed nanoparticles. Furthermore, there is also a need for the development and implementation of techniques that offer in situ information of the ligand shell, such as small angle neutron scattering (SANS) [60] and quartz crystal microbalance with dissipation monitoring [61].

To-date, only few reports have demonstrated the long-term robustness of flow synthesis by monitoring the reactor output over representative operating times. Instead, the common practice is to limit the operation of the flow reactor to relatively short times until a steady state is reached to collect enough sample for characterization.

One of the biggest advantages of flow reactors, the high surface-to-volume ratio, can lead to one of the biggest challenges connected to their usage in the field of nanomaterials synthesis: fouling. The accumulation of particles on the reactor walls not only reduces the synthesis yield, but also changes the synthesis conditions during operation. Fouling is in general a dynamic process, rendering the operation of the flow system dynamic rather than continuous. Segmenting liquids that preferentially wet the reactor wall are a common solution to fouling (as well as improved residence time distribution) [62]. However, while such approaches may efficiently avoid contact of the reaction solution with the reactor wall, the nanoparticle quality can suffer from interfacial adsorption [63]. 
Flow reactors present particular advantages for the use of gaseous reactants, where tuning of the system pressure and efficient mass transfer can be achieved more effectively in comparison to batch reactors. Examples in this context are the synthesis of AuNPs of various sizes and surface functionalities in gas-liquid segmented flow reactors, gas-liquid-liquid and membrane liquid-liquid reactors [64], [65], and the synthesis of atomically precise $\mathrm{Au}_{25}$ cluster in membrane liquid-liquid reactors [66], where in all cases $\mathrm{CO}$ was used as reducing agent.

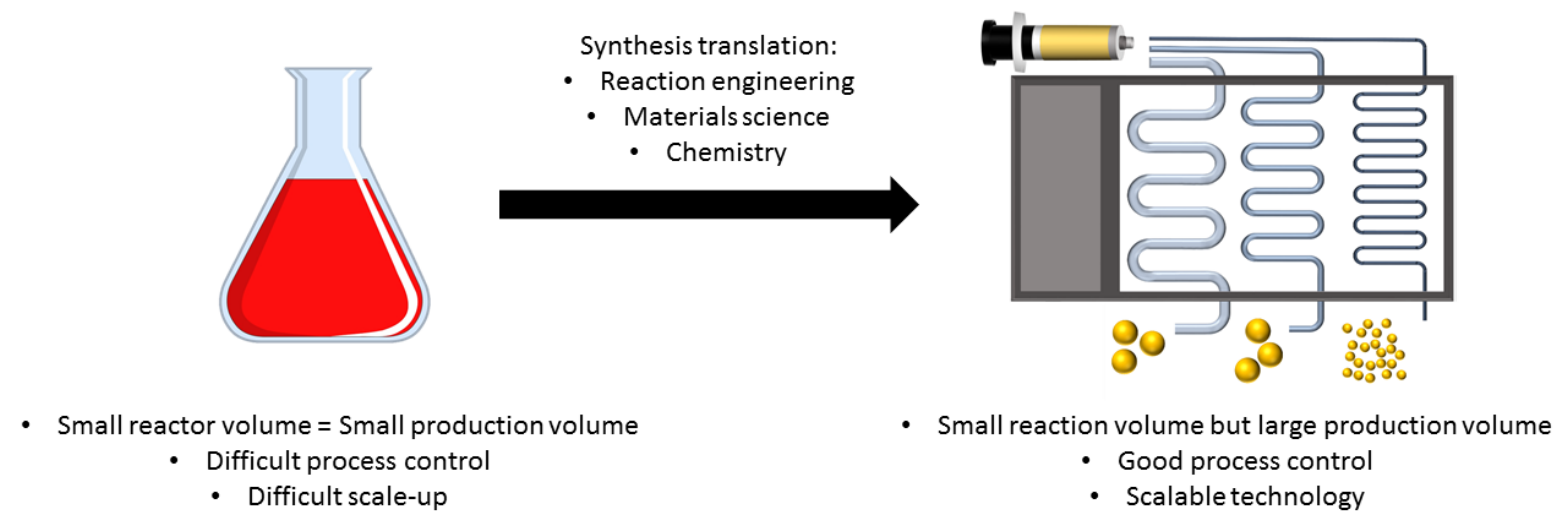

Figure 4: Translation of gold nanoparticles synthesis from batch to flow. A number of advantages derive from the use of flow reactor, though the efficient translation of the synthesis from batch to flow requires a significant interdisciplinary effort. Adapted with permission from reference [67] under Creative Commons CC-BY license. Copyright 2018 Elsevier.

Flow reactors offer the opportunity for controlled and scalable manufacturing of gold nanoparticles through available batch protocols up to $\mathrm{g} \mathrm{Au} /$ day productivities [68], [69]. Some examples are already available in the literature addressing scale up of the production: Huang et al. scaled-up the production of Au25 clusters compared to the equivalent batch synthesis reported in the literature by increasing the precursor concentration by a factor of 10 , increasing the reaction temperature and decreasing the reaction time from $24 \mathrm{~h}$ to only $3 \mathrm{~min}$, while retaining full precursor conversion as in the equivalent batch synthesis [63]. In this way the production of approximately $1 \mathrm{~g}$ of Au25 clusters per day was achieved, with a continuous operation of the reactor over 3 days without deviation in the particles properties. Lohse et al. reported the shape-controlled synthesis of Au nanomaterials with productivity in the order of $2.5 \mathrm{mg} / \mathrm{min}$ (which projected over $24 \mathrm{~h}$ operation corresponds to $3.6 \mathrm{~g} /$ day) [68]. Gomez et al. scaled-up the production of Au nanocages both in batch and flow, demonstrating that the maximum achievable yield (without compromising the product quality) in batch was $115 \mathrm{~g} / \mathrm{day}$, against $\sim 570 \mathrm{~g} /$ day achievable in flow [70].

Optimal design of these reactors requires an interdisciplinary effort linking chemistry, material science and engineering. Research efforts are still required for a straightforward translation of existing batch protocols to flow, in particular to find innovative ways to fight fouling and to investigate synthesis kinetics [57]. The unique advantages of flow over batch reactors (e.g., operation at higher temperature/pressure, fast heating/cooling, short reaction times, controlled staged addition of reactants) are only starting to be leveraged and require the development of suitable synthetic protocols.

\section{Summary and future directions}

Recent developments on the engineering of gold nanoparticle synthesis provide pathways to scaledup production of nanoparticles, with reproducible properties with respect to narrow size distribution, 
uniform shape and surface functionality. We anticipate the following aspects to be key points for further progress in the near future:

a) While considerable improvements have been reported to-date, full control over the batch-tobatch reproducibility remains challenging. In this respect, detailed control over the nucleation kinetics and implementation of protocols that promote the decoupling of nucleation and growth are crucial. A more synergistic interplay between batch and flow synthesis may help to resolve underlying fundamentals and enable to adapt the most suitable synthetic approach for the respective application and design targets.

b) Flow technologies offer attractive opportunities towards both fundamental understanding of synthetic protocol, as well as nanomaterials manufacturing. Scalability of these technologies is still challenging, hence we anticipate that in the near future the best opportunities for flow technologies implementation in industrial settings will be in the production of high-value nanomaterials for biomedical applications, where the production scale is relatively small (below $1000 \mathrm{~kg} /$ year) compared to other industrial sectors.

c) In order to ensure reliable nanomaterial synthesis in flow, more robust design procedures are required, in particular to ensure full conversion of the precursor at the reactor outlet, as unreacted precursor can both hinder control over the size distribution and lower the process yield. New and more robust design procedures should be informed by principles of reaction engineering and statistics (e.g. model based design of experiments). The latter requires more effort in implementing inline analysis techniques that can provide data in real time, eventually allowing the design of closed-loop and self-optimizing systems. Furthermore, in order to make flow processes attractive from a commercial point of view, emphasis needs to be put on the implementation of downstream purification processes, which are currently generally overlooked or performed in batch-mode (e.g. centrifugation).

d) The field of atomically precise Au clusters is rapidly gaining interest, in particular in the field of biomedicine. These provide significant advantages, both with respect to their efficient use of material as well as their ability for renal clearance.

\section{Acknowledgements}

This project received funding from the European Union's Horizon 2020 research and innovation programme under grant agreement no. 633635 (DIACHEMO). LP received funding from the European Union's Horizon 2020 research and innovation programme under the Marie Skłodowska-Curie grant agreement $\mathrm{N}^{\circ} 721290$. This publication reflects only the authors' view, exempting the community by any liability. Project website: http://cosmic-etn.eu/. MOB and AG would like to thank the EPSRC for financial support (grant EP/M015157/1) through the Manufacturing Advanced Functional Materials (MaFuMa) scheme.

\section{References and recommended reading}

1 Sun J, Xianyua Y, Jiang X: Point-of-care biochemical assays using gold nanoparticle-implemented microfluidics. Chem Soc Rev 2014, 43:6239-6253.

2.De la Rica R, Stevens MM: Plasmonic ELISA for the ultrasensitive detection of disease biomarkers with the naked eye. Nature Nanotechnology 2012, 7:821-824.

3. Huang X, Aguilar ZP, Xu H, Lai W, Xiong Y: Membrane-based lateral flow immunochromatographic strip with nanoparticles as reporters for detection: a review. Biosensors and Bioelectronics 2016, 75:166-180.

4. Donati P,Moglianetti M, Veronesi M, Prato M, Tatulli G, Bandiera T, Pompa PP: Nanocatalyst/nanoplasmonenabled detection of organic mercury: a one-minute visual test. Angew Chem Int Ed 2019, 58:10285-10289. 5. Macdonald TJ, Wu K, Sehmi SK, Noimark S, Peveler WJ, du Toit H, Voelcker NH, Allan E, MacRobert AJ, Gavriilidis A, Parkin IP: Thiol-capped gold nanoparticles swell-encapsulated into polyurethane as powerful antibacterial surfaces under dark and light conditions. Scientific Reports 2016, 6:39272. 
6. Zhou N, López-Puente V, Wang Q, Polavarapu L, Pastoriza-Santos I, Xu Q-H: Plasmon-enhanced light harvesting: applications in enhanced photocatalysis, photodynamic therapy and photovoltaics. RSC Advances 2015, 5:29076-29097.

7. You CC, Miranda OR, Gider B, Ghosh PS, Kim IB, Erdogan B, Krovi SA, Bunz UHF, Rotello VM: Detection and identification of proteins using nanoparticle-fluorescent polymer chemical nose' sensors. Nat. Nanotechnol. 2007, 2:318-323.

8. Gabrielli L, Rosa-Gastaldo D, Salvia M-V, Springhetti S, Rastrelli F, Mancin F: Detection and identification of designer drugs by nanoparticle-based NMR chemosensing. Chem Sci 2018, 9:4777-4784.

9. Gupta A, Mumtaz S, Li C-H, Hussain I, Rotello V M: Combatting antibiotic-resistant bacteria using nanomaterials. Chemical Society Reviews 2019, 48:415-427.

** Comprehensive overview of surface engineering strategies for AuNPs as antimicrobial agents.

10. Cagno $V$ et al.: Broad-spectrum non-toxic antiviral nanoparticles with a virucidal inhibition mechanism. Nature Materials 2017, 17:195-203.

**Important study in the emerging field of antiviral nanoparticles.

11. Guo Q, Xu M, Yuan Y, Gu R, Yao J: Self-Assembled Large-Scale Monolayer of Au Nanoparticles at the Air/Water Interface Used as a SERS Substrate. Langmuir 2016, 32:4530-4537.

12.Lynachan CN, Soleimany AP, Dudani JS, Lin Y, Najer A, Bekdemir A, Chen Q, Bhatia SN, Stevens MM: Renal clearable catalytic gold nanoclusters for in vivo disease monitoring. Nature Nanotechnology 2019, 14:883890.

**Innovative study highlighting novel avenues for in-vivo applications of gold nanoparticles.

13. Bodelón G, Costas C, Pérez-Juste J, Pastoriza-Santos I, Liz-Marzán LM: Gold nanoparticles for regulation of cell function and behaviour. Nano Today 2017, 13:40-60.

14. Nakhleh MK et al.: Diagnosis and classification of 17 diseases from 1404 subjects via pattern analysis of exhaled molecules. ACS Nano 2017, 11:112-125.

15. a) https://aurion.nl; b) https://bbisolutions.com; c) https://blacktrace.com d) http://exicuretx.com/; e) https://hiqnano.com f) http://midatechpharma.com; g) https://nanoimmunotech.com; h)

https://nanocomposix.com; i) https://vsparticle.com;

16. Mahapatra I, Sun TY, Clark JRA, Dobson PJ, Hungerbuehler K, Owen R, Nowack B, Lead J: Probabilistic modelling of prospective environmental concentrations of gold nanoparticles from medical applications as a basis for risk assessment. J Nanobiotechnol 2015, 13:93.

17. Dreaden EC, Alkilany AM, Huang X, Murphy CJ, El-Sayed MA: The golden age: gold nanoparticles for biomedicine. Chem. Soc. Rev. 2012, 41:2740-2779.

18. Dykman LA, Khlebtsov NG: Uptake of engineered gold nanoparticles into mammalian cells. Chemical Reviews 2014, 114:1258-1288.

19 Jiang Y, Huo S, Mizuhara T, Das R, Lee Y-W, Hou S, Moyano DF, Duncan B, Liang X-J, Rotello VM: The interplay of size and surface functionality on the cellular uptake of sub-10 $\mathbf{n m}$ gold nanoparticles. ACS Nano 2015, 9:9986-9993.

20 Marson D, Yang Y, Guldin S, Posocco P: Noble metal nanoparticles with anisotropy in shape and surface functionality for biomedical applications. Anisotropic Particle Assemblies 2018, 313-333.

21. Grzelczak M, Pérez-Juste J, Mulvaney P, Liz-Marzán LM: Shape control in gold nanoparticle synthesis. Chem. Soc. Rev. 2008, 37:1783-1791.

22. Zhaoc P, Li N, Astruc D: State of the art in gold nanoparticle synthesis. Coordination Chemistry Reviews 2013, 257: 638-665.

23. Baer DR: The chameleon effect: characterization challenges due to the variability of nanoparticles and their surfaces. Front Chem 2018, 6:145-152.

24. Ji X, Song X, Li J, Bai Y, Yang W, Peng X: Size control of gold nanocrystals in citrate reduction: the third role of citrate. J Am Chem Soc 2007, 129:13939-13948.

25. Bastus NG, Comenge J, Puntes V: Kinetically controlled seeded growth synthesis of citrate-stabilized gold nanoparticles of up to $200 \mathrm{~nm}$ : size focusing versus Ostwald ripening. Langmuir 2011, 27:11098-11105.

26. Brust M, Walker M, Bethell D, Schiffrin DJ, Whyman R J: Synthesis of Thiol-Derivatized Gold Nanoparticles in a Twophase, Liquid-Liquid System. J Chem Soc 1994, 7:801-802.

27. Brust M, Fink J, Bethell D, Schiffrin D J, Kiely CJ: Synthesis and Reactions of Functionalised Gold Nanoparticles. J Chem Soc 1995, 16:1655-1656.

28. Zheng N, Fan J, Stucky GD: One-step one-phase synthesis of monodisperse noble-metallic nanoparticles and their colloidal crystals. J Am Chem Soc 2006, 128:6550-6551

29. Peng S, Lee $Y$, Wang $C$, Yin H, Dai S, Sun S: A facile synthesis of monodisperse au nanoparticles and their catalysis of co oxidation. Nano Res 2008, 1:229-234.

30. Luo Z, Yuan X, Zhang Q, Leong DT, Lee, JY, Xie J: From aggregation-induced emission of au(i)-thiolate complexes to ultrabright au(0)@au(i)-thiolate core-shell nanoclusters. J.A.C.S. 2012, 134:16662-16670. 
31. Yu Y, Chen X, Yao Q, Yu Y, Yan N, Xie J: Scalable and precise synthesis of thiolated Au10-12, Au15, Au18, and Au25 nanoclusters via pH controlled CO reduction. Chemistry of Materials 2013, 25:946-952.

32. Yu Y, Luo Z, Yu Y, Lee JY, Xie J: Atomically precise clusters of noble metals: emerging link between atoms and nanoparticles. Chemical Reviews 2017, 117: 8208-8271.

33. Piella J, Bastús NG, Puntes V: Size-controlled synthesis of sub-10-nanometer citrate-stabilized gold nanoparticles and related optical properties. Chem. Mater. 2016, 28:1066-1075.

* Synthetic breakthrough, enabling access to sub-10 $\mathrm{nm}$ nanoparticles by citrate chemistry.

34. Yao Q, Yuan X, Fung V, Yu Y, Leong DT, Jiang D, Xie J: Understanding seed-mediated growth of gold nanoclusters at molecular level. Nature Commun 2017, 8:927.

35. O'Brien MN, Jones MR, Brown KA, Mirkin CA: Universal noble metal nanoparticle seeds realized through iterative reductive growth and oxidative dissolution reactions. J Am Chem Soc 2014, 136:7603-7606.

** Insights in the nanoparticle iterative seed refinement describing its structural control and characterization. 36. Biju V: Chemical modifications and bioconjugate reactions of nanomaterials for sensing, imaging, drug delivery and therapy. Chem Soc Rev 2014,43:744-764.

*Comprehensive summary of gold nanoparticles formation and surface addressing for specific interaction.

37 Luo Z, Hou J, Menin L, Ong QK, Stellacci F: Evolution of the ligand-shell morphology during ligand-exchange reaction on gold nanoparticles. Angewandte Chemie - International Edition 2017, 16:13521-13525.

* Detailed study on the evolution of the ligand shell composition and morphology during the thiol-for-thiol ligand exchange synthesis using the emerging MALDI-TOF technique.

38. Brown LO, Hutchison JE: Convenient preparation of stable, narrow-dispersity, gold nanocrystals by ligand exchange reactions. J Am Chem Soc 1997, 119:12384-12385.

39 Gittins DI, Caruso F: Spontaneous phase transfer of nanoparticulate metals from organic to aqueous media. Angewandte Chemie - International Edition 2001, 17:3001-3004.

40. Manea F, Bindoli C, Polizzi S, Lay L, Scrimin P: Expeditious synthesis of water-soluble, monolayer-protected gold nanoparticles of controlled size and monolayer composition. Langmuir 2008, 24:4120-4124.

41. Mourdikoudis S, Liz-Marzán LM: Oleylamine in nanoparticle synthesis. Chem Mater 2013, 25:1465-1476.

42. Yang Y, Serrano LA, Guldin S: A Versatile AuNP Synthetic Platform for Decoupled Control of Size and

Surface Composition. Langmuir 2018, 34:6820-6826.

** Versatile synthetic protocol enabling full control over both size and ligand shell of sub-10 nm gold nanoparticles.

43. Yu M, Xu J, Zheng J: Renal clearable luminescent gold nanoparticles: from the bench to the clinic. Angew. Chem. Int. Ed. 2019, 58:4112-4128.

44. Polte J, Erler R, Thünemann AF, Sokolov S, Ahner TT, Rademann K, Emmerling F, Kraehnert R: Nucleation and growth of gold nanoparticles studied via in situ small angle $x$-ray scattering at millisecond time resolution. ACS Nano 2010, 4:1076-1082.

45. Wuithschick M, Birnbaum A, Witte S, Sztucki M, Vainio U, Pinna N, Rademann K, Emmerling F, Kraehnert R, Polte J: Turkevich in new robes: key questions answered for the most common gold nanoparticle synthesis. ACS Nano 2015, 9:7052-7071.

* Rigorous study revealing mechanistic insights into the fundamental principles involved in gold nanoparticle synthesis by citrate reduction.

46. Thanh NTK, Maclean N, Mahiddine S: Mechanisms of nucleation and growth of nanoparticles in solution. Chem Rev 2014, 114:7610-763.

47. Besenhard MO, Baber R, LaGrow AP, Mazzei L, Thanh NTK, Gavriilidis A: New insight into the effect of mass transfer on the synthesis of silver and gold nanoparticles. Cryst Eng Comm 2018, 20:7082-7093.

*Study elucidating the importance of mass transfer in determining the size and degree of polydispersity in batch synthesis of gold nanoparticles.

48. Huang H, du Toit H, Panariello L, Mazzei L, Gavriilidis A: 4. Continuous synthesis of gold nanoparticles in micro- and millifluidic systems. Metallic Nanomaterials (Part A) 2018, 157-220, Berlin, Boston: De Gruyter. Taifur-Rahman M, Rebrov E. Microreactors for gold nanoparticles synthesis: from Faraday to flow. Processes 2014, 2:466-493.

49. Elvira KS, Casadevall i Solvas X, Wootton RCR, DeMello AJ: The past, present and potential for microfluidic reactor technology in chemical synthesis. Nature Chemistry 2013, 5:905-915.

50. Panariello L, Damilos S. du Toit H, Wu G, Radhakrishnan ANP, Parkin IP, Gavriilidis A: Highly reproducible, high-yield flow synthesis of gold nanoparticles based on a rational reactor design exploiting the reduction of passivated Au(III). React. Chem. Eng. 2020.

51. Panariello L, Mazzei L, Gavriilidis A: Modelling the synthesis of nanoparticles in continuous microreactors: The role of diffusion and residence time distribution on nanoparticle characteristics. Chemical Engineering Journal 2018, 350: 1144-1154.

52. Perala SRK, Kumar S: On the mechanism of metal nanoparticle synthesis in the Brust-Schiffrin method. Langmuir 2013, 31:9863-9873. 
53. Agunloye E, Panariello L, Gavriilidis A, Mazzei L: A model for the formation of gold nanoparticles in the citrate synthesis method. Chemical Engineering Science 2018, 191: 318-331.

54. Kettemann F, Birnbaum A, Witte S, Wuithschick M, Pinna N, Kraehnert R, Rademann K, Polte J: Missing piece of the mechanism of the turkevich method: the critical role of citrate protonation. Chemistry of Materials 2016, 28:4072-4081.

55. Schulz F, Homolka T, Bastús NG, Puntes V, Weller H, Vossmeyer T: Little adjustments significantly improve the turkevich synthesis of gold nanoparticles. Langmuir 2014, 35:10779-10784.

56. Chen X, Schröder J, Hauschild S, Rosenfeldt S, Dulle M, Förster S: Simultaneous SAXS/WAXS/UV-VIS study

of the nucleation and growth of nanoparticles: a test of classical nucleation theory. Langmuir 2015, 31:1167811691.

57. Polte J, Erler R, Thünemann AF, Sokolov S, Ahner TT, Rademann K, Emmerling F, Kraehnert: Nucleation and growth of gold nanoparticles studied via in situ small angle $x$-ray scattering at millisecond time resolution. ACS Nano 2010, 4:1076-1082.

58. Mourdikoudis S, Pallares RM, Thanh NTK: Characterization techniques for nanoparticles: comparison and complementarity upon studying nanoparticle properties. Nanoscale 2018, 27:12871-12934.

59. Hendel T, Wuithschick M, Kettemann F, Birnbaum A, Rademann K, Polte J: In situ determination of colloidal gold concentrations with uv-vis spectroscopy: limitations and perspectives. Analytical Chemistry 2014, 86:11115-11124.

60. Luo Z, Yang Y, Radulescu A, Kohlbrecher J, Darwish TA, Ong QK, Guldin S, Stellacci F: Multidimensional characterization of mixed ligand nanoparticles using small angle neutron scattering. Chemistry of Materials 2019, 31:6750-6758.

61. Yang Y, Poss G, Weng Y, Qi R, Zheng H, Nianias N, Kay ER, Guldin S: Probing the interaction of nanoparticles with small molecules in real time via quartz crystal microbalance monitoring. 2019, 23:11107-11113.

62. Panariello L, Mazzei L, Gavriilidis A: Modelling the synthesis of nanoparticles in continuous microreactors: The role of diffusion and residence time distribution on nanoparticle characteristics. Chemical Engineering Journal 2018, 350: 1144-1154.

63. Zhang L, Wang Y, Tong L, Xia Y: Synthesis of colloidal metal nanocrystals in droplet reactors: the pros and cons of interfacial adsorption. Nano Letters 2014, 14:4189-4194.

64. Huang H, du Toit H, Ben-Jaber S, Wu G, Panariello L, Thi Kim Thanh N, Parkin IP, Gavriilidis A: Rapid synthesis of gold nanoparticles with carbon monoxide in a microfluidic segmented flow system. React Chem Eng 2019, 4:884-890.

65. Rahman T, Krishnamurthy PG, Parthiban P, Jain A, Pil Park C, Kim D-P, Khan SA: Dynamically tunable nanoparticle engineering enabled by short contact-time microfluidic synthesis with a reactive gas. $R S C$ Advances 2013,3:2897-2900.

66. Huang H, Byoung Hwang G, Wu G, Karu K, Du Toit H, Wu H, Callison J, Parkin IP, Gavriilidis A: Rapid synthesis of [Au25(CYS)18] nanoclusters via carbon monoxide in microfluidic liquid-liquid segmented flow system and their antimicrobial performance. Chemical Engineering Journal 2019, 123176.

*High throughput fast synthesis of pure Au25 clusters using gaseous reactants in a membrane reactor with good antimicrobial properties.

67. Huang H, Du Toit H, Besenhard MO, Ben-Jaber S, Dobson P, Parkin IP, Gavriilidis A: Continuous flow synthesis of ultrasmall gold nanoparticles in a microreactor using trisodium citrate and their SERS performance. Chemical Engineering Science 2018, 189:422-430.

68. Lohse SE, Eller JR, Sivapalan ST, Plews MR, Murphy CJ: A simple millifluidic benchtop reactor system for the high-throughput synthesis and functionalization of gold nanoparticles with different sizes and shapes. ACS Nano 2013, 7:4135-4150.

**First report of high throughput synthesis of multiple gold nanostructures in the same millifluidic reactor. 69. Murphy CJ, Lohse SE, Eller JR: Continuous flow reactor and method for nanoparticle synthesis. US patent US9375790B2 2013.

70. Leyre G, Sebastian V, Irusta S, Ibarra A, Arruebo M, Santamaria J: Scaled-up production of plasmonic nanoparticles using microfluidics: from metal precursors to functionalized and sterilized nanoparticles. Lab Chip 2014, 14:325-332. 